\title{
SemanPhone: Combining Semantic and Phonetic Word Association in Verbal Learning Context
}

\author{
Lu Jiyan \\ School of Software and \\ Microelectronics \\ NorthWestern Polytechnical \\ University \\ Xi'an, China \\ martinahill@outlook.com \\ Susanna Pirttikangas \\ Center for Ubiquitous Computing \\ University of Oulu \\ Oulu, Finland \\ susanna.pirttikangas@oulu.fi
}

\author{
Panos Kostakos \\ Center for Ubiquitous Computing \\ University of Oulu \\ Oulu, Finland \\ panos.kostakos@oulu.fi
}

\author{
Mourad Oussalah \\ Center for Ubiquitous Computing \\ University of Oulu \\ Oulu, Finland \\ mourad.oussalah@oulu.fi
}

\begin{abstract}
This paper proposes an effective way to discover and memorize new English vocabulary based on both semantic and phonetic associations. The method we proposed aims to automatically find out the most associated words of a given target word. The measurement of semantic association was achieved by calculating cosine similarity of two-word vectors, and the measurement of phonetic association was achieved by calculating the longest common subsequence of phonetic symbol strings of two words. Finally, the method is implemented as a web application.
\end{abstract}

Keywords- Word association, Semantic similarity, Phonetic similarity, WordNet, GloVe, Verbal learning.

\section{INTRODUCTION}

The Graduate Record Examinations (GRE) is a general standardized test that is being used as a typical academic entry requirement in most graduate schools in the United States. Experiences in preparing for GRE test for non-English speakers have pointed out to the challenges of memorizing thousands of English words in a relatively short time [1]. The connection between words through either semantic meaning, morphological transformation or phonetic connection is a well-known phenomenon. This raises the question on how to efficiently use word-association for the purpose of boosting word-memorizing and vocabulary-building capabilities. Word associations are prevalent heuristics that date back to Aristotle's four Classical Laws of Association [2] stating that mental items (ideas, perceptions, sensations, or feelings) are connected in memory under the following conditions: i) they occur simultaneously ("spatial contact"); ii) they occur in close succession ("temporal contact"); iii) they are similar; iv) they are contrary.

Craik and Lockhart's levels of processing (LOP) model views memory for verbal stimuli as a function of encoding [3]. The level of encoding assigned to each word determines its memorability. When shallow processing occurs, only superficial aspects are encoded. Respectively, deeper level of encoding takes extra time to process the semantic meaning of the stimulus but the formed memory would persist longer.

In the same vein, Rohwer (1966) found that children who studied noun pairs in sentence contexts (e.g., "The cow kicked the ball") had higher recall of "ball" when later cued with "cow" than subjects (Ss) who studied just the two nouns (e.g., "cow ball") [4]. In trying to extend Rohwer's findings, Bobrow and Bower (1969) found that college Ss who were instructed to generate their own sentences linking two nouns had higher cued noun recall than Ss who studied a sensible sentence containing the same nouns [5]. That is, the S who studied "farmer diamond" by creating the sentence, "The farmer found a diamond in his field," had greater recall of "diamond" when cued with "farmer" than the control $\mathrm{S}$ who simply read a provided sentence such as, "The farmer found a diamond."

Strictly speaking, verbal linguistic research highlighted three main strategies for memorizing vocabulary: repetition, contextualization and association. Through memory stimulus mechanism, repetition is the simple form of memorizing largely adopted in schools. Contextualization refers to putting the target word in a context to better comprehend its meaning using the discourse aspect. This turns to be useful for foreign language learners in input-poor environments [6]. Association relies on the fact that ideas and concepts available in our memory are inherently related. Thus, the activation of one idea or concept is accompanied by the activation of other ideas or concepts correlated with it. This phenomenon is called the association [7]. Although repetition and contextualization, are simple to obtain, association between words is rather more challenging. This motivates the current work undertaken in this paper contributing to the challenge of automatic discovery of words which are both semantically and phonetically associated with the given word.

Therefore, for a given keyword, the key problem we are trying to tackle consists in finding the most prominent words which are both semantically and phonetically associated with that given keyword. For instance, for the keyword "ascribe", which means "to consider that sth is caused by a particular thing or person", it will be efficient to return the word "assign" as one of prominent words, which means "to give sth to sb". Because these two words not only sound similar, but also have the semantic meaning involving "giving" something to somebody.

With a growing need for non-native English speakers to perform well in standardized test, several applications have been put forward to help learners in word memorization process. Table 1 lists out some related application according to their performance and core functionalities. 
TABLE I. OVERVIEW OF RELATED APPLICATIONS

\begin{tabular}{|c|c|c|c|c|c|c|}
\hline Application & D & $\mathbf{M}$ & $\mathbf{W}$ & Speed & Memory & Function \\
\hline Visuwords [8] & & & $\mathrm{x}$ & medium & medium & $\begin{array}{l}\text { graphical } \\
\text { dictionary }\end{array}$ \\
\hline OneLook [9] & & & $\mathrm{x}$ & high & medium & $\begin{array}{l}\text { thesaurus/ } \\
\text { reverse } \\
\text { dictionary }\end{array}$ \\
\hline $\begin{array}{l}\text { RhymeZone } \\
\text { [10] }\end{array}$ & & & $\mathrm{x}$ & high & better & $\begin{array}{l}\text { various-single- } \\
\text { filtering } \\
\text { association } \\
\text { dictionary }\end{array}$ \\
\hline $\begin{array}{l}\text { Microsoft } \\
\text { Bing } \\
\text { Dictionary } \\
{[11]}\end{array}$ & $\mathrm{x}$ & $\mathrm{x}$ & $x$ & high & medium & $\begin{array}{l}\text { general } \\
\text { dictionary and } \\
\text { ideographic } \\
\text { dictionary }\end{array}$ \\
\hline SemanPhone & & & $\mathrm{x}$ & high & better & $\begin{array}{l}\text { double- } \\
\text { filtering } \\
\text { association }\end{array}$ \\
\hline
\end{tabular}

D: desktop; M: mobile; W: web.

This paper advocates a new approach for word association that combines the semantic, morphological, and contextual aspect together with phonetic aspect. A web application called SemanPhone can be accessed on semanphone.fun server enabling the user to test the developed algorithm on a selected target word as well as record their feedback for further processing. The application has been implemented as a server like application and tested over a population of Chinese students. The usability and feasibility of the proposal has been demonstrated through an online questionnaire. Section 2 of this paper highlights the various stages of the developed methodology. Section 3 exhibits the implementation, exemplification and some results. Finally, conclusive statements and perspective work are reported in Section 4.

\section{METHOD}

\section{A. General Approach}

In the ocean of English words, it is obvious that only a small portion of them are having relations with a given target word, so there is no need to perform pairwise comparisons with every single word of English dictionary. Therefore, it is rational to initially seek constructing a set of relatively large candidate words, which will be next refined, yielding a much small set of candidate words.

To achieve this, metrics based on semantic association and phonetic association are elaborated, and then combined in order to derive a comprehensive indicator that screens the "best" words out of candidate word set. The overall procedure is summarized in the following four-step strategy:

Step 1: Construct a smaller candidate words set from all English words.

Step 2: Apply a method to measure semantic association.

Step 3: Apply a method to measure phonetic association.

Step 4: Combine the above two measurements to screen out "best" words from candidate set.

\section{B. Candidate Word Set Generation}

Given a target word, an intuitive way to identify potentially semantical or morphologically related words is through exploring already existing dictionary or word ontology, e.g., WordNet ${ }^{1}$. The latter is a large lexical database of English. Nouns, verbs, adjectives and adverbs are grouped into sets of cognitive synonyms (synsets), each expressing a distinct concept. Synsets are interlinked by means of conceptual-semantic and lexical relations. For instance, words which are linked to the target word can be established by observing WordNet associations (e.g., synonymy, antonymy, hyponymy and hypernymy). On the other hand, other online dictionaries/repositories are also potential sources for extracting related words.

For this purpose, we used Datamuse ${ }^{2}$ API in order to explore word associations beyond WordNet lexical database limitation. This can be achieved with Datamuse API because it queries a large number of online repositories and search engines. While the implementation details underneath the Datamuse API is not fully known, dozens of online dictionaries crawled by OneLook in addition to WordNet are reported to have been employed by the API.

\section{ALGORITHM I. GETCANDIDATESET(WORD)}

input: Target word

output: candidate words associated to target word

\section{candidate set $\leftarrow$ null}

$\mathrm{q} \leftarrow$ query (data source $=$ Datamuse, filter condition $=$ means like words $)$

candidate set $\leftarrow$ candidate set $+\mathrm{q}$

central synsets $\leftarrow$ get all synsets of word from WordNet

For synset in central synsets do

candidate set $\leftarrow$ candidate set + word in synset

candidate set $\leftarrow$ candidate set + word in hyponym synsets of synset

candidate set $\leftarrow$ candidate set + word in hypernym synsets of synset

candidate set $\leftarrow$ candidate set + word in sibling synsets of synset

End for

return candidate set

On the other hand, our initial testing has revealed that associated words extracted using WordNet embedded relation has shown little overlap with that outputted by Datamuse API. This supports the idea of useful hybridization between the two generated associated words. More formally, for a given target word, we collect words from all synsets which contain the word that a user wishes to memorize. Secondly, we also collect words from all hypernym synsets, all hyponym synsets and all sibling synsets of the central synsets.

As a consequence, we will utilize WordNet and Datamuse to form this candidate set of associated words. The process of generating candidate words set is shown in Algorithm 1. Gathering words from Datamuse API is intuitive, while gathering from WordNet requires a little more effort. As shown in Figure 1, every synset (i.e. node in taxonomy) is linked to other synsets.

\section{Semantic Association}

Given the large candidate words generated by Algorithm 1, using Datamuse API together with WordNet lexical relations, the key challenge is to use appropriate metric in order to reduce this set to a sufficiently small number items

\footnotetext{
${ }^{1}$ WordNet: https://wordnet.princeton.edu
}

${ }^{2}$ The Datamuse API: http://www.datamuse.com/api/ 
that is easy to convey and interact with potential learners. For this purpose, both the semantic similarity and phonetic association will be employed.

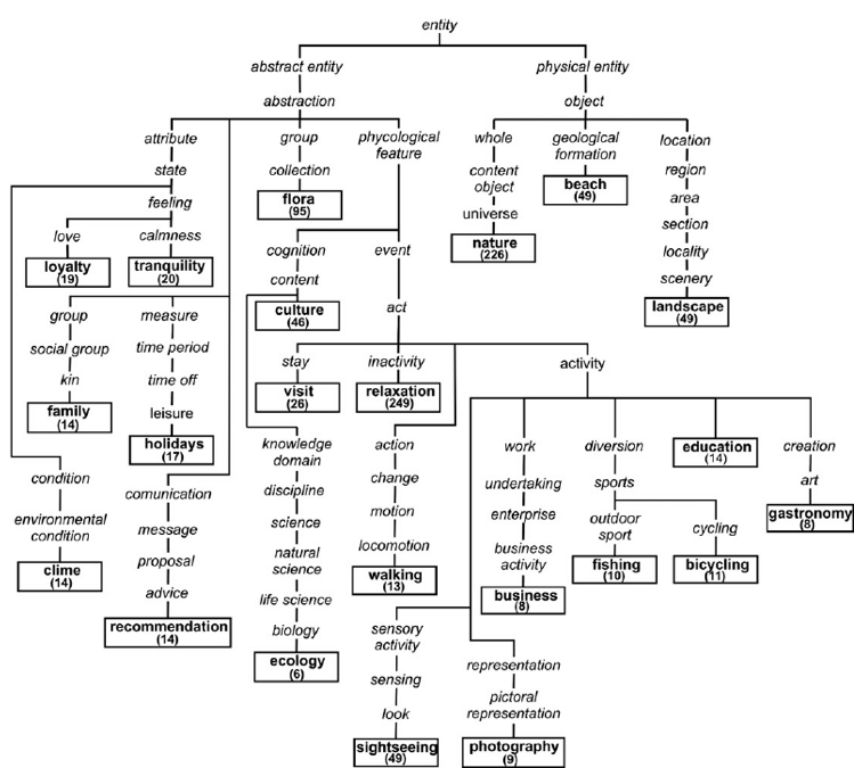

Fig. 1. Taxonomy structure of WordNet for various datasets (in bold). Source: [12]

\section{1) Semantic similarity metric}

In the context of semantic similarity metric, one distinguishes at least three distinct approaches. The first type, often referred to as Mutual Information, is based on cooccurrence rate only. Namely, for every pair of words $P$ and $\mathrm{Q}$, we count the number of times word $\mathrm{P}$ occurs in a corpus $\mathrm{P}(\mathrm{P})$, the number of times word $\mathrm{Q}$ occurs in a corpus $\mathrm{P}(\mathrm{Q})$ and the number of times word $\mathrm{P}$ and word $\mathrm{Q}$ co-occur in a corpus $\mathrm{P}(\mathrm{P}, \mathrm{Q})$ to calculate the Mutual Information Value of $\mathrm{P}$ and $\mathrm{Q}$ to measure their semantic similarity, e.g., see [13].

The second type combines corpus statistics and lexical taxonomy. The taxonomy structure was considered into the model by calculating the shortest path from one node containing word $P$ to another containing word $Q$. Cooccurrence probability counted from corpus was considered into the model as Information Content Value of the closest common node which indicates how much word $P$ and word $Q$ have in common, e.g., [14].

The third one leverages the power of web search engine as performed in [15]. It is also based on corpus statistic data but the innovation is that it shrinks the counting range by selecting only related data source using web search engine. The focus in on new counting objects like page count and extracted snippets instead of the whole corpus.

The final type makes of deep-learning models: GloVe and Word2Vec. They both learn their word vectors from cooccurrence statistics of words. GloVe trained word vectors using Global Log Bilinear Regression Model [16] while Word2Vec using Neural Network. More information about the learning process of Word2Vec can be found in [17]. One can use these models to train vectors as well as using their trained vectors, both trained from very sufficient data source. The latter approach is adopted in our study. This is motivated by the fact that first approach lacks a predefined open large database for word occurrence count, which makes it less practical to our case. The second one is flexible because it reached a relatively balanced point by combining useful information from two aspects, but it is also requiring a readyto-use large database of Information Content Value for all nodes in the taxonomy. Due to the fact that we already took advantage of WordNet taxonomy structure in our previous step (constructing the candidate set), it lost part of attraction of its combination of corpus statistics and lexical taxonomy. The third method is heavily dependent on the search engine configuration and, most importantly, it requires pre-prepared patterns to extract snippets to be used. Among GloVe and Word2Vec, decided to choose GloVe because of its proven efficiency on word similarity tasks as reported in [16]. Having two-word vectors, one corresponding to the target word and the other one to candidate word generated by Algorithm 1, we define the Semantic Association Value as Cosine Value between the two word vectors. The higher the cosine value, the higher is the word association accordingly.

Sem_Ass_Value $(\mathrm{T}, \mathrm{Q})=$ Cosine $(\operatorname{GloVe}(\mathrm{T}), \operatorname{GloVe}(\mathrm{Q}))$

\section{2) Phonetic association}

The idea behind the phonetic association relies on the intuition that if a word can be brought to your mind via another word because of their pronunciation, these two words are phonetically associated.

Pronunciation of a word is determined by its phonetic symbols, which we can obtain by looking up the word in CMU Pronouncing Dictionary ${ }^{3}$ which is an open-source and machine readable pronunciation dictionary containing over 134,000 words. The phonetic symbol is actually a string, consisting of characters in ARPAbet ${ }^{4}$ set. So, measuring similarity between pronunciation of two words can be converted to measuring two phonetic symbol strings. For simplicity and practicality, we use phonetic string for phonetic symbol string. For this purpose, we first find the Longest Common Subsequence of the two phonetic strings, as described in Algorithm 2. The motivation for using the Longest Common Subsequence instead of the commonly employed Longest Common Substring is exemplified through the following pair of words:

$$
\text { "thirty" and "thirsty" }
$$

If we were to find the longest common substring, we can only capture "thir", but actually two words sound similar more than this truncation as they have another common part "ty" which also gives out the same pronunciation. By taking the longest common subsequence, we can find out both "thir" and "ty", and the result better reflects the relationship of the two words, which motivates the choice of Longest Common Subsequence as our comparing strategy.

Besides, looking at the basic comparison unit in phonetic strings whether it is a single character, or a syllable (consisting of a bunch of characters) the length of basic comparison unit varies. For example, this can be illustrated by comparing phonetic strings of "cut" and "cat".

$[\mathrm{K}, \mathrm{AH} 1, \mathrm{~T}]$ (the number 1 in AE1 indicates lexical stress)

$[\mathrm{K}, \mathrm{AE} 1, \mathrm{~T}]$

\footnotetext{
${ }^{4}$ https://en.wikipedia.org/wiki/ARPABET
}

\footnotetext{
${ }^{3}$ http://www.speech.cs.cmu.edu/cgi-bin/cmudict
} 
We start by comparing the first element in [K, AH1, T] with the first element in [K, AE1, T] we get the first common element and put it in common subsequence [K]. Then, we continue by comparing the second element in [K, AH1, T]. $\mathrm{AH} 1$, with the second element in [K, AE1, T], AE1, they are considered different terms even though they have two same characters. After a series of comparisons, we got the final longest common subsequence, $[\mathrm{K}, \mathrm{T}]$. Through this example we can see that the basic comparison unit can be either a single character sometimes (like $\mathrm{K}$ and $\mathrm{T}$ ) or a group of several characters (like AH1 and AE1). In such case, the basic comparison unit reflects the basic unit of uncombined pronunciation directly.

In Algorithm 2, we define the Phonetic Association Value between two phonetic strings as two times of length of common part divided by the sum of length of two strings. Though very simple, it still has good performance in distinguishing similar sound words from non-similar sound words.

\section{AlgorithM II. COMPUTEPHONETICASSOCIATION(TARGET WORD CANDIDATE WORD)}

input: two words

output: phonetic association value of two words

first phonetic string $\leftarrow$ GetPhoneticSymbol(target word)

second phonetic string $\leftarrow$ GetPhoneticSymbol(candidate word)

first len $\leftarrow$ length(first phonetic string)

second len $\leftarrow$ length(second phonetic string)

common subsequen $\leftarrow$ LongestCommonSubsequence(first phonetic string, second phonetic string)

common len $\leftarrow$ length(common subsequence)

phonetic association value $\leftarrow 2 *$ (common len $) /($ first len + second len $)$

return phonetic association value

\section{3) Integrate metric}

Our model advocates a convex combination between the semantic association value and the phonetic association value. More specifically,

Integrated_Ass_Value $=\alpha *$ Phonetic_Ass_Value

$+(1-\alpha) *$ Sem_Ass_Value

The background is that all words in the candidate words set are more or less semantically related to the target word. Thus, our final goal is to find out those words which sound like the target word and are as similar as possible. So, from our perspective, phonetic association value should contribute more to the integrated association value, as we already considered semantic factors in the previous step so this time semantic factor is no longer the primary role, as a consequence $\boldsymbol{\alpha}$ should be a value between $(0.5,1)$

Although, the choice of the trade-off parameter $\alpha$ is debatable and open to discussion, the parameter was determined through replicable experimentation (details of the set-up are omitted for brevity).

\section{EXEMPLFICATION AND IMPLEMENTATION}

We have implemented SemanPhone as a web application that can be accessed on semanphone.fun. The application is an
English word association explorer and not an online dictionary. Users can use it to discover words which are highly associated both semantically and phonetically with a given keyword.

Figure 2 shows a use case of the application for the word "partake". The user types the word "partake" into the search bar, clicks RETURN, then then gets five words which SemanPhone thinks are highly associated with "partake". At this point, the user will probably learn the meaning of the word "partake" via the help of "participate" -assuming that the meaning of the word "participate" is already known to the user- because they have similar meaning as well as similar pronunciation. The complete source code is available in Github $^{5}$.

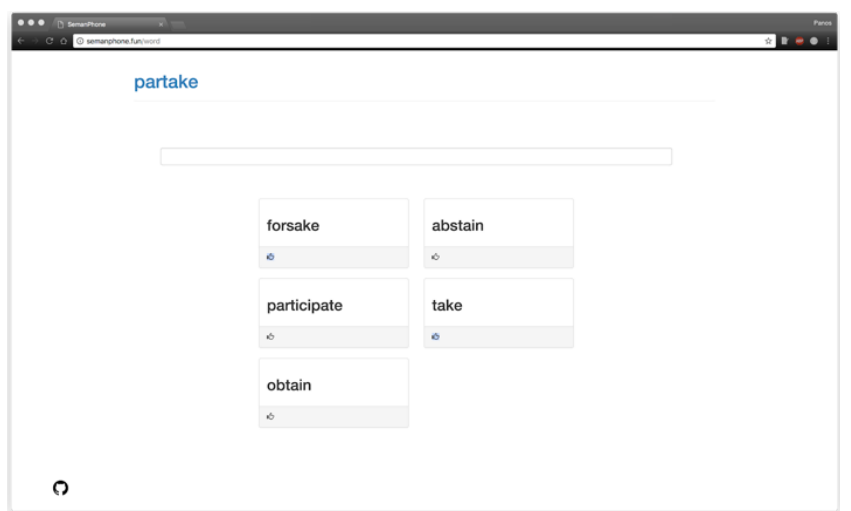

Fig. 2. Implementation of the Application

In order to test how effective SemanPhone is in helping memorizing new words, we invited some test volunteers by spreading test invitation link on social network inside NorthWestern Polytechnical University in China. So, test volunteers are mainly consisted of university students and their friends, most of them are at the age between 18 and 30, receiving higher education, and having vocabulary size between 5000 to 15000 . We design the following experiment to measure the performance of the application:

1. When a tester queries a target word in the search bar (shown in Figure 2), she/he will get five associated words back by SemanPhone.

2. If she/he thinks any of the returned words is helpful to memorize the target word, she/he would mark that word as a useful word by clicking a like button under that word. If a target word has any of its returned words marked useful by any tester, we think SemanPhone has produced helpful results for that target word.

3. Our website would record every target word searched by testers, as well as all the words which have been marked useful by testers.

4. Finally, we count the total number of distinct target words all testers have queried, mark it as T. We also count the number of distinct target words for which SemanPhone has produced helpful results, mark it as $\mathrm{H}$. Then, we calculate the proportion of $\mathrm{H}$ over T, and take this proportion as being the app Performance.

If performance is more than $50 \%$, we assume SemanPhone works as expected, because for more than $50 \%$ of queried

\footnotetext{
${ }^{5}$ https://github.com/MartiBook/SemanPhone
} 
target words, SemanPhone has produced useful results for our user. Actually, we cannot expect Performance index to reach $100 \%$ for two reasons. First, some English words may not have such associated words following our definition at all. Second, some users may have a small vocabulary size which makes them sometimes not able to judge associations between words.

\section{A. Results}

Figure 3 depicts the database for the collected raw test data and query statements needed to retrieve information. We have two tables to record raw test data. The left column shows the content in table query, it records words which have been queried by tester and the query time of each word. The right column shows the content of recommendation table, it records recommended words (returned results of our application), the time of that word being marked useful by testers, and the query word for which this word is recommended.

We have 863 distinct words queried, and 548 of them have their returned results marked useful by test volunteers. We got a Performance of $\mathbf{6 3 . 5 0 \%}$, which shows our application works as expected. Table 2 shows some examples that we think SemanPhone did a good job.

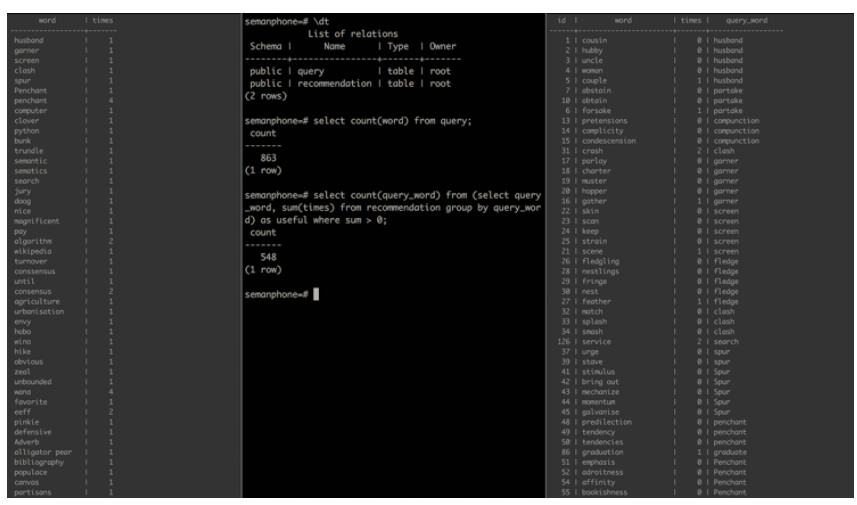

Fig. 3. Database of collected user test data

\section{CONCLUSION}

Learning and remembering new words can be a challenging task. We have designed, implemented, and validated an application that enables users to learn new words based on the word association heuristic. In fact, our application not only enables users to discover possible associated words to help them boost memory retrieval capabilities, but also offers new words from vocabulary already known to the user/learner. Users can actually perform reverse engineering by inputting a word already know and retrieving new associations.

The first step was to collect candidate words and take full advantage of existing the WordNet lexical database and Datamuse API. This saved a great deal of resources by constraining our candidate set. We took both semantic and phonetic factors into account when measuring the word association. Although a convex combination is used to tradeoff the two components, further research will be required in order to comprehend a more contextual based consensus aggregation. Especially, experiments of the $\alpha$ value could be expanded into using larger sample data, adopting training models, and also possibly allowing a self-evolving parameter which can be revised along user queries.

Finally, we think we can inject some self-taught ability into our application by adding some user interaction features. For example, users should be able to vote up and vote down any of returned words, and every voting up and voting down should be considered to rearrange returned words when the same word is searched next time. Similarly, users should be able to add or remove words to or from the returned word list. Our application will take these actions into account when providing search results for the same word next time.

\section{TABLE II. ASSOCIATED WORDS FOUND BY SEMANPHONE}

\begin{tabular}{|l|l|l|l|}
\hline \multicolumn{1}{|c|}{$\begin{array}{c}\text { Target } \\
\text { word }\end{array}$} & $\begin{array}{c}\text { The best returned } \\
\text { word }\end{array}$ & \multicolumn{1}{|c|}{$\begin{array}{c}\text { Target } \\
\text { word }\end{array}$} & $\begin{array}{c}\text { The best } \\
\text { returned word }\end{array}$ \\
\hline recap & Recall & stumble & tumble \\
\hline amicable & amiable & didactic & academic \\
\hline garner & Gather & adulation & adoration \\
\hline penchant & Passion & endemic & domestic \\
\hline hodgepodge & mishmash & sardonic & sarcastic \\
\hline undermine & undercut & magisterial & masterful \\
\hline overbearing & domineering & profess & Confess \\
\hline incursion & invasion & lol & huh \\
\hline
\end{tabular}

\section{ACKNOWLEDGMENTS}

This work is (partially) funded by the European Commission grant 770469-CUTLER and 645706-GRAGE

\section{REFERENCES}

[1] ETS (2017). GRE Worldwide Test Taker Report - July 2012-June 2017

https://www.ets.org/s/gre/pdf/snapshot_test_taker_data_2017.pdf

[2] Kohonen, T. (2012). Self-organization and associative memory (Vol. 8). Springer Science \& Business Media.

[3] Craik, F., Lockhart, R. "Levels of processing: A framework for memory research",Journal of Verbal Learning and Verbal Behavior,11(6): 671-684, 1972.

[4] Rohwer, W.D. Constraint, syntax and meaning in paired-associate learning, Journal of Verbal Learning and Verbal Behavior, 1966, 5, S41-S47.

[5] Bobrow, S.A and Bower, G.H. "Comprehension and recall of sentences", Journal of experimental Psychology, 80(30): 455-461.

[6] Gu, Y., \& Johnson, R. K. (1996). Vocabulary learning strategies and language learning outcomes. Language learning, 46(4), 643-679.

[7] V. V. Morkovkin. (1972). Ideographic Dictionaries. Moscow, USSR

[8] https://visuwords.com/

[9] https://www.onelook.com/

[10] https://www.rhymezone.com/

[11] https://www.microsoft.com/en-us/p/microsoft-bing-dictionarychinese-english/9nblggh63sw0

[12] Martínez, S., Sánchez, D. and Valls, A. "Semantic adaptive microaggregation of categorical microdata", Computers \& Security,31(5): 653-672,2012

[13] Church, K. W., \& Hanks, P. (1990). Word association norms, mutual information, and lexicography. Computational linguistics, 16(1), 2229. 
[14] Jiang, J. J., \& Conrath, D. W. (1997). Semantic similarity based on corpus statistics and lexical taxonomy. arXiv preprint cmp-lg/9709008.

[15] Bollegala, D., Matsuo, Y., \& Ishizuka, M. (2007). Measuring semantic similarity between words using web search engines. www, 7, 757-766.

[16] Pennington, J., Socher, R., \& Manning, C. (2014). Glove: Global vectors for word representation. In Proceedings of the 2014 conference on empirical methods in natural language processing (EMNLP) (pp. 1532-1543)

[17] Rong, X. (2014). word2vec parameter learning explained. arXiv preprint arXiv:1411.2738. 